\title{
BMJ Global Health Projections of human papillomavirus (HPV) vaccination impact in Ethiopia, India, Nigeria and Pakistan: a comparative modelling study
}

\author{
Allison Portnoy (D , , Kaja Abbas (D , ${ }^{2,3}$ Steven Sweet, ${ }^{1,4}$ Jane J Kim, ${ }^{1}$ \\ Mark Jit (1) 2,5
}

To cite: Portnoy A, Abbas K, Sweet S, et al. Projections of human papillomavirus (HPV) vaccination impact in Ethiopia, India, Nigeria and Pakistan: a comparative modelling study. BMJ Global Health 2021;6:e006940. doi:10.1136/ bmjgh-2021-006940

Handling editor Seye Abimbola

Received 19 July 2021

Accepted 18 0ctober 2021
Check for updates

(c) Author(s) (or their employer(s)) 2021. Re-use permitted under CC BY. Published by BMJ.

${ }^{1}$ Center for Health Decision Science, Harvard University T H Chan School of Public Health, Boston, Massachusetts, USA ${ }^{2}$ Department of Infectious Disease Epidemiology, London School of Hygiene \& Tropical Medicine, London, UK

${ }^{3}$ Public Health Foundation of India, New Delhi, India

${ }^{4}$ Vitalant Research Institute, San Francisco, California, USA

${ }^{5}$ School of Public Health, The University of Hong Kong, Hong Kong, Hong Kong

Correspondence to Dr Allison Portnoy; aportnoy@mail.harvard.edu

\section{ABSTRACT}

Introduction Cervical cancer is the second most common cancer among women in Ethiopia, India, Nigeria and Pakistan. Our study objective was to assess similarities and differences in vaccine-impact projections through comparative modelling analysis by independently estimating the potential health impact of human papillomavirus (HPV) vaccination.

Methods Using two widely published models (Harvard and Papillomavirus Rapid Interface for Modelling and Economics (PRIME)) to estimate HPV vaccination impact, we simulated a vaccination scenario of $90 \%$ annual coverage among 10 cohorts of 9-year-old girls from 2021 to 2030 in Ethiopia, India, Nigeria and Pakistan. We estimated potential health impact in terms of cervical cancer cases, deaths and disability-adjusted life years averted among vaccinated cohorts from the time of vaccination until 2100 . We harmonised the two models by standardising input data to comparatively estimate HPV vaccination impact

Results Prior to harmonising model assumptions, the range between PRIME and Harvard models for number of cervical cancer cases averted by HPV vaccination was: 262000 to 270000 in Ethiopia; 1640000 to 1970000 in India; 330000 to 336000 in Nigeria and 111000 to 133000 in Pakistan. When harmonising model assumptions, alignment on HPV type distribution significantly narrowed differences in vaccine-impact estimates.

Conclusion Despite model differences, the Harvard and PRIME models yielded similar vaccine-impact estimates. The main differences in estimates are due to variation in interpretation around data on cervical cancer attribution to HPV-16/18. As countries make progress towards WHO targets for cervical cancer elimination, continued explorations of underlying differences in model inputs, assumptions and results when examining cervical cancer prevention policy will be critical.

\section{INTRODUCTION}

Persistent infections with human papillomavirus (HPV) types 16 and 18 cause $70 \%$ of all cases of cervical cancer. ${ }^{12}$ Studies have

\section{Key questions}

What is already known?

- Studies have shown that prophylactic human papilIomavirus (HPV) vaccination provides almost 100\% protection against persistent infection with vaccinetargeted high-risk HPV strains (eg, HPV-16, 18) and associated precancers if administered prior to sexual initiation.

- Health-impact modelling has been used to project vaccination impact and estimate the timeline to eliminate cervical cancer as a public health problem (ie, reducing country-level annual cervical cancer incidence to below 4 per 100000 ).

What are the new findings?

- The differences in outcomes between the models capture variation in interpretation around data on cervical cancer epidemiology and future demographic change.

- This study highlights that HPV-type distribution is a critical input to modelling the potential health impact of vaccination.

What do the new findings imply?

- In order to accelerate progress towards cervical cancer elimination, preventing cervical cancer through HPV vaccination will be an essential strategy, particularly given low coverage and access to cervical cancer screening in low-income and middle-income country settings.

- Understanding similarities and differences between HPV vaccination impact predicted by different models will be crucial, given that key questions about which countries to prioritise and which vaccination strategies to use will be important in an era of HPV vaccine dose shortages and COVID-19-related disruptions to vaccination programmes.

shown that prophylactic HPV vaccination provides almost $100 \%$ protection against persistent infection with vaccine-targeted high-risk HPV strains (eg, HPV-16, 18) and associated precancers if administered prior to 
sexual initiation. ${ }^{34}$ The WHO has set a goal to eliminate cervical cancer as a public health problem by 2100 , which involves reducing country-level annual cervical cancer incidence to below 4 per $100000 .^{5-7}$ However, vaccine coverage remains low in low-income and middle-income countries (LMICs) that also lack high-quality screening programmes. $^{8}$ More than $85 \%$ of cervical cancer deaths occur in $\mathrm{LMICs}^{9}$ with cervical cancer being the leading cause of female cancer death in sub-Saharan Africa. ${ }^{10}$

Cervical cancer imposes the second greatest burden of cancer incidence among women in Ethiopia, India, Nigeria and Pakistan as well as the second greatest burden of cancer mortality among women in Ethiopia, India and Nigeria-and the fourth greatest burden of cancer mortality among women in Pakistan. ${ }^{11}$ The International Agency for Research on Cancer (IARC) estimated that 148000 new cases occurred and 94000 women died from cervical cancer in these four countries in $2020 .{ }^{11}$ However, of these four countries, only Ethiopia has implemented nationwide HPV vaccination, with a single-age cohort campaign of 14-year-old girls in 2018-2019, ${ }^{8}{ }^{12}$ while India has introduced it in a few states in $2016 .{ }^{13}$

Most new vaccine introductions in those countries have been supported through partnerships with the global community, particularly Gavi, the Vaccine Alliance, and the Bill and Melinda Gates Foundation (BMGF). Mathematical models of the impact of HPV vaccines have been used to support programme monitoring and priority setting by Gavi and BMGF since 2011. ${ }^{14}$ The Vaccine Impact Modelling Consortium (VIMC) was formed in late 2016, with the support of Gavi and BMGF, to bring together two groups involved in HPV modelling, with others conducting impact modelling of 11 other vaccines. ${ }^{15}$ Additionally, by ensuring that at least two groups model each analysed pathogen within VIMC, the vaccine-impact estimates from VIMC provide an important opportunity to examine the parametric, structural, model and methodological uncertainty both within and between models. ${ }^{16}$ Comparative modelling aims to enhance model transparency and can help guide public health research and priorities.

The two HPV vaccine models in VIMC are the Papillomavirus Rapid Interface for Modelling and Economics (hereafter PRIME), developed by a consortium of modellers led by the London School of Hygiene \& Tropical Medicine and the Harvard model (hereafter Harvard), developed by a team of modellers at the Harvard T.H. Chan School of Public Health. Both models have been used extensively to inform decisions by Gavi, ${ }^{17}{ }^{18} \mathrm{BMGF}^{19}$ $\mathrm{WHO}^{20-22}$ and individual countries. ${ }^{2324}$ Given that both models are being used to understand HPV vaccine impact in the same settings, it is important to quantitatively compare the projections made by both models and to understand their differences, so that model results can be interpreted in the context of each other.

Our study objective was to assess similarities and differences in vaccine-impact projections through comparative modelling analysis by independently estimating the potential health impact of HPV vaccination among 10 cohorts of 9-year-old girls from 2021 to 2030 in Ethiopia, India, Nigeria and Pakistan. We used a vaccination scenario of $90 \%$ annual coverage among 9-year-old girls, in alignment with the goals of the cervical cancer elimination strategy set forth by the WHO. ${ }^{5-7}$ We estimated the potential health impact in terms of cervical cancer cases, deaths and disability-adjusted life years (DALYs) averted among vaccinated cohorts from the time of vaccination until 2100 in Ethiopia, India, Nigeria and Pakistan. We conducted a comparative modelling analysis to infer the differences in the vaccine-impact estimates generated by the PRIME and Harvard models.

\section{METHODS}

We used the PRIME and Harvard models to project the impact of HPV vaccination in four high-burden countries (Ethiopia, India, Nigeria and Pakistan). Both the PRIME $^{22} 25$ and Harvard ${ }^{172}$ models have been extensively described and validated elsewhere; we summarise their main features below.

\section{Model overview}

Both the Harvard and PRIME models are static, multicohort, proportional impact models that can estimate the impact of HPV vaccination on cervical cancer cases and deaths. The models estimate vaccination impact in terms of reductions in age-dependent cervical cancer incidence and mortality in direct proportion to vaccine efficacy against HPV-16/18, vaccine coverage and HPVtype distribution.

The models assume that girls are fully immunised with a two-dose schedule with perfect timeliness at the target ages and that girls effectively immunised against vaccine-targeted HPV types can develop cervical cancer associated with non-vaccine HPV types; also, neither crossprotection against non-vaccine types nor indirect effects are assumed. The models capture burden from all HPV genotypes, but the impact of vaccination is limited to the burden caused by genotypes targeted by the vaccine. In this analysis, the models simulated health benefits from vaccination against HPV types 16 and 18 . Vaccine efficacy against HPV-16/18 infections is assumed to be $100 \%^{26-30}$ over the lifetime. Herd effects are not considered, so the vaccine-impact estimates produced are conservative. The models assume that age-specific cervical cancer incidence among unvaccinated women remains constant over the time horizon of the model.

\section{Data sources}

Table 1 outlines the data sources used by the Harvard and PRIME models. Age-specific cervical cancer incidence is estimated from the database of IARC. ${ }^{11}$ For the proportion of cancer that is attributed to the vaccinecovered types (eg, HPV-16/18), PRIME uses a study by Serrano $e t a l^{31}$ whose data sources include a meta-analysis performed by IARC $^{32}$ and a retrospective cross-sectional 
Table 1 Data sources and overview of comparative analysis for Harvard and PRIME models

\begin{tabular}{|c|c|c|}
\hline Feature & Harvard & PRIME \\
\hline Model structure & Proportional outcomes & Proportional outcomes \\
\hline Population representation & Open, multi-cohort & Open, multi-cohort \\
\hline Representation of cancer progression & $\begin{array}{l}\text { Country-specific distributions of } \\
\text { cancer stages, assuming } 2 \text { years lived } \\
\text { with disability and } 5 \text { years survival } \\
\text { for individuals experiencing cancer } \\
\text { mortality }{ }^{21}\end{array}$ & $\begin{array}{l}\text { Based on Global Burden of Disease- } \\
\text { prescribed durations and phases (diagnosis } \\
\text { and primary treatment phase, controlled } \\
\text { phase, metastatic phase and terminal } \\
\text { phase) }\end{array}$ \\
\hline Disability-adjusted life year estimation & $\begin{array}{l}\text { Based on weighted averages of Global } \\
\text { Burden of Disease-prescribed weights } \\
\text { for Stages I-III and Stage IV } \text { IV }^{35}\end{array}$ & $\begin{array}{l}\text { Based on Global Burden of Disease- } \\
\text { prescribed weights }^{35}\end{array}$ \\
\hline Cervical cancer incidence & Globocan 2020 database of IARC ${ }^{11}$ & Globocan 2020 database of IARC ${ }^{11}$ \\
\hline Cervical cancer prevalence & Not applicable & Globocan 2020 database of IARC ${ }^{11}$ \\
\hline Population size & $\begin{array}{l}\text { United Nations World Population } \\
\text { Prospects } 2019 \text { estimates }^{36}\end{array}$ & $\begin{array}{l}\text { United Nations World Population Prospects } \\
2019 \text { estimates combined with time- } \\
\text { varying, country-specific probability of } \\
\text { death for projected estimates }{ }^{36}\end{array}$ \\
\hline Life tables & $\begin{array}{l}\text { World Health Organization } 2019 \text { life } \\
\text { tables (constant) }{ }^{37}\end{array}$ & $\begin{array}{l}\text { Constructed with United Nations } \\
\text { Population Division time-varying, country- } \\
\text { specific probability of death }\end{array}$ \\
\hline HPV-16/18 proportion & Meta-analysis by IARC ${ }^{32}$ & $\begin{array}{l}\text { Serrano et } a^{31} \text { based on meta-analysis by } \\
\text { IARC }^{32} \text { and retrospective cross-sectional } \\
\text { study }^{33}\end{array}$ \\
\hline
\end{tabular}

HPV, human papillomavirus; IARC, International Agency for Research on Cancer; PRIME, Papillomavirus Rapid Interface for Modelling and Economics.

worldwide study ${ }^{33}$ while Harvard uses the meta-analysis by IARC $^{32}$ exclusively.

To estimate cancer mortality, the Harvard model assumes country-specific distributions of cancer stages. ${ }^{21}$ The model then incorporates 5-year stage-specific survival probabilities for untreated and treated cervical cancers (by region) and treatment access proportions (by country). These values are combined into weighted averages to provide country-specific 5-year survival parameters by stage, validated against age-specific mortality rates. ${ }^{11} 21$ The PRIME model uses estimates of age-specific cervical cancer mortality from Globocan $2020 .^{11}$

In the Harvard model, disability weights are assumed to be 0.2 for stages I-III cervical cancer and 0.4733 for stage IV cervical cancer, based on the Global Burden of Disease (GBD) studies, ${ }^{34} 35$ and all cervical cancer cases experienced an average of 2 years lived with disability. In PRIME, disability weights are also based on GBD studies for the different phases of cervical cancer: diagnosis and primary treatment phase $(0.288)$, controlled phase (0.049), metastatic phase $(0.451)$ and terminal phase $(0.540)$. The disability weights and durations for the different phases of cervical cancer are used in estimating the years of life lost due to disability.

\section{Demography}

PRIME is a multiple cohort model. It calculates population size by estimating the size of the female age cohort at the age of vaccination (eg, 9 years old) from World Population Prospects 2019 estimates. ${ }^{36}$ The size of the age cohort in subsequent years is then calculated by constructing life tables, using the time-varying probability of dying by age and country from the World Population Prospects 2019 estimates. ${ }^{36}$

In contrast, the Harvard model is a population-based model that uses only data from the World Population Prospects 2019 estimates. ${ }^{36}$ The base year (eg, 2010) is used for the population projections in year 0 , the next year $(\mathrm{eg}, 2011)$ is used for the population projections in year 1 and so forth. Life tables from the WHO are used for calculating DALYs, but not for population projections. Demographic estimates for age-specific population size (in 1-year intervals) and age-specific life expectancy (in 5-year intervals) were from United Nations World 
Population Prospects 2019 estimates $^{36}$ and 2019 WHO life tables ${ }^{37}$ respectively. In years when no data were available, we used a growth factor calculated as a function of a country's population.

\section{Vaccination scenarios}

We conducted analyses to evaluate the impact of HPV vaccination assuming $90 \%$ coverage of annual, routine vaccination of 9-year-old girls vaccinated in 2021-2030 (ie, ten cohorts). We assumed $100 \%$ protection against HPV-16 and 18 infections over the lifetime of vaccines for a two-dose vaccination schedule.

\section{Model outcomes}

Cervical cancer cases, deaths and DALYs averted were calculated in comparison with a strategy of no HPV vaccination in the four high-burden countries (Ethiopia, India, Nigeria and Pakistan) using both PRIME and Harvard models. Model outcomes were aggregated over multiple birth cohorts to capture the health benefits of vaccinating girls aged 9 years between 2021 and 2030 from the time of vaccination until 2100.

\section{Comparative analysis}

The principles of our comparative modelling study are: (1) addressing policy questions on HPV vaccination impact, (2) selection of two widely published models (Harvard and PRIME) to estimate HPV vaccination impact, (3) harmonisation of input data (demography, prevaccination cervical cancer burden and HPV 16/18type distribution) and outputs (cervical cancer cases, deaths and DALYs), (4) exploring variability in estimates of HPV vaccination impact within and between Harvard and PRIME models, (5) presenting the comparative results and (6) projecting lifetime health impact among adolescent girls at $90 \%$ vaccination coverage (a key objective of the global cervical cancer elimination strategy). ${ }^{38}$ This aligns with the principles presented in the guidelines for multimodel comparisons of the impact of infectious disease interventions. ${ }^{39}$

Model inputs, including population demography and HPV-16/18-type distribution, were harmonised in order to evaluate model differences. Harmonisation refers to standardisation of input data and outputs between multiple models to address a research question. ${ }^{39}$ In our case, we standardised input data (demography, prevaccination cervical cancer burden, and HPV 16/18-type distribution) and outputs (cervical cancer cases, deaths and DALYs) in the Harvard and PRIME models to compare the projections in HPV vaccination impact (cases, deaths and DALYs averted by vaccination).

\section{Patient and public involvement}

Patients and the public were not involved in this study.

\section{RESULTS \\ Descriptive model differences}

Figure 1A shows the differences between the data used for the proportion of cervical cancer that is attributed to HPV-16/18 by the two models for Ethiopia, India, Nigeria and Pakistan. Figure 1B compares the cohort size for a cohort born in 2012 (which relates to the vaccinated cohort of 9-year-old girls in 2021) in the two models using Pakistan as an example; all four countries showed similar differences and trends. While, on average, the differences in cohort size range from $2 \%$ to $6 \%$, the differences remain small until older ages (80 years and above), when the differences increased due to the decreasing population size with increasing all-cause mortality.

\section{Cervical cancer cases, deaths and DALYs averted}

Under different assumptions for HPV-16/18-type distribution and demography, the Harvard model estimated a greater number of cervical cancer cases averted than the PRIME model by 3\% in Ethiopia, 20\% in India, 2\% in Nigeria and $19 \%$ in Pakistan (figure 2A). Specifically, the range between the PRIME model and the Harvard model for the potential health impact of HPV vaccination in terms of the number of cervical cancer cases averted among girls vaccinated in 2021-2030 between the year of vaccination and 2100 was: 262000 to 270000 in Ethiopia; 1640000 to 1970000 in India; 330000 to 336000 in Nigeria and 111000 to 133000 in Pakistan.

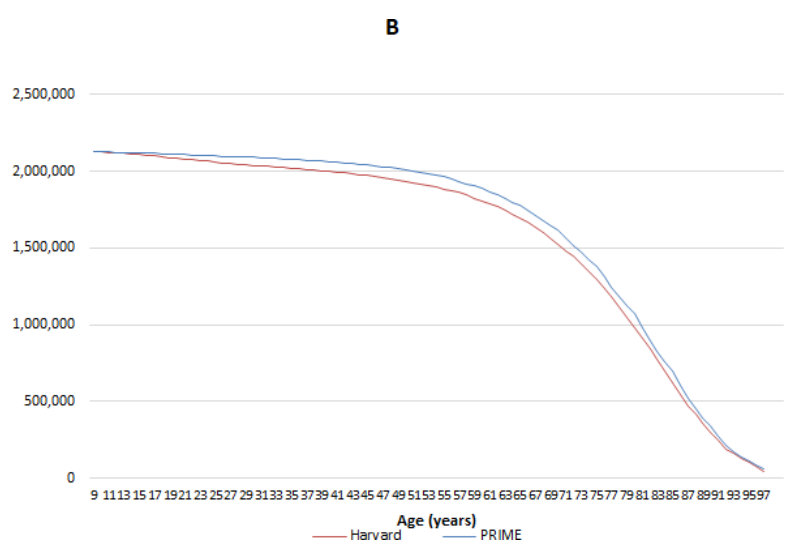

Figure 1 (A) Proportion of cervical cancer attributable to HPV-16/18; and (B) population size over time for 9-year-old girls born in 2012 (vaccinated in 2021) in Pakistan. HPV, human papillomavirus; PRIME, Papillomavirus Rapid Interface for Modelling and Economics. 


\section{A}
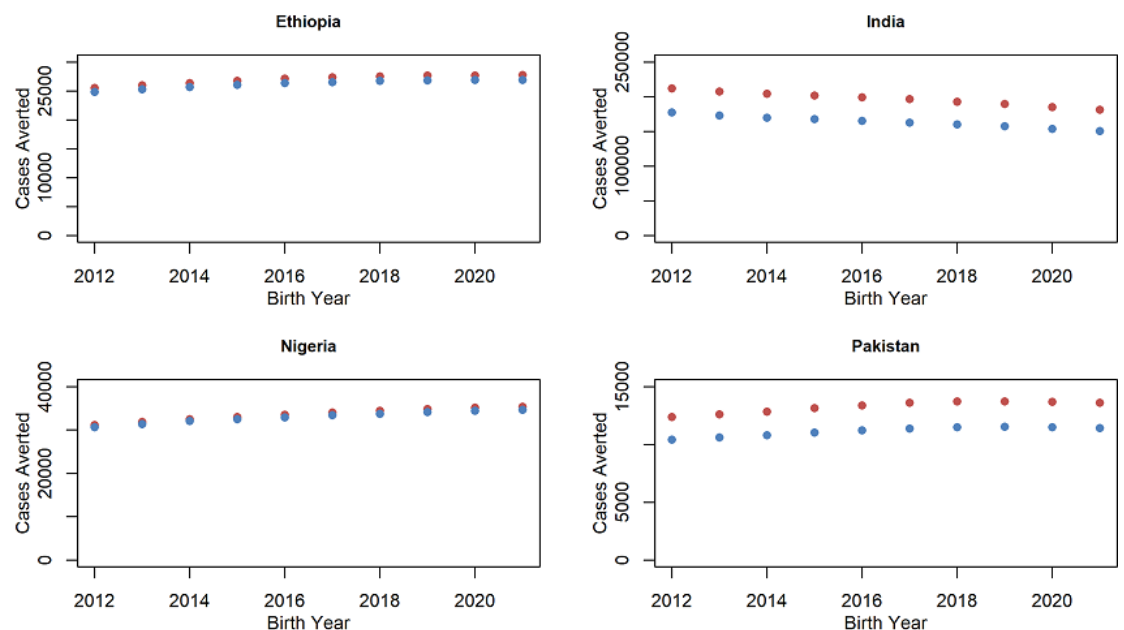

B
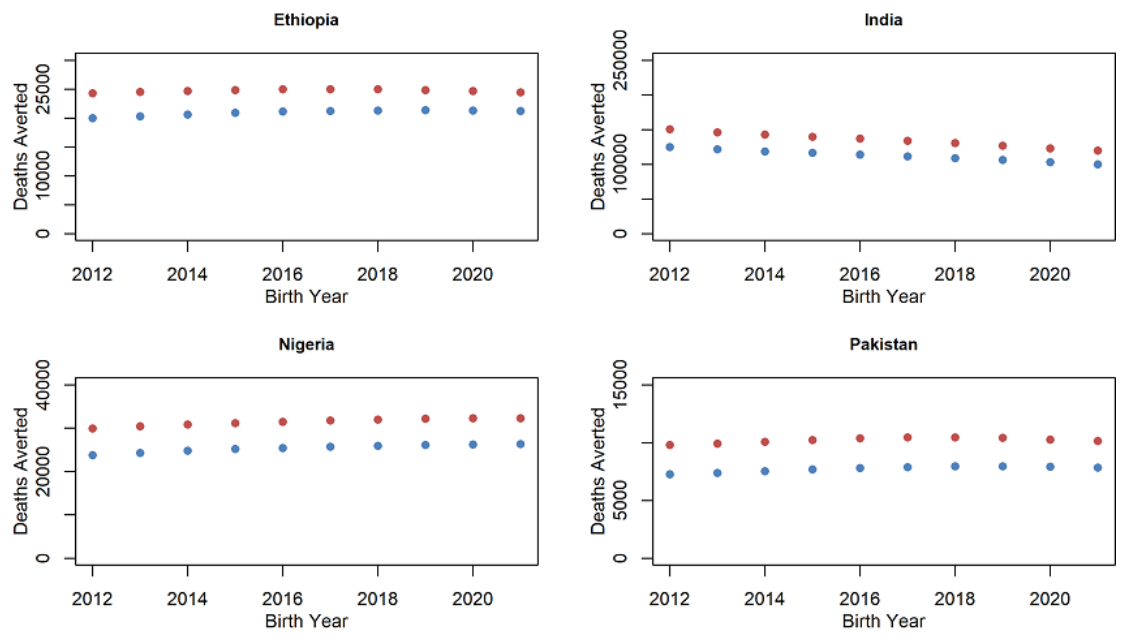

C
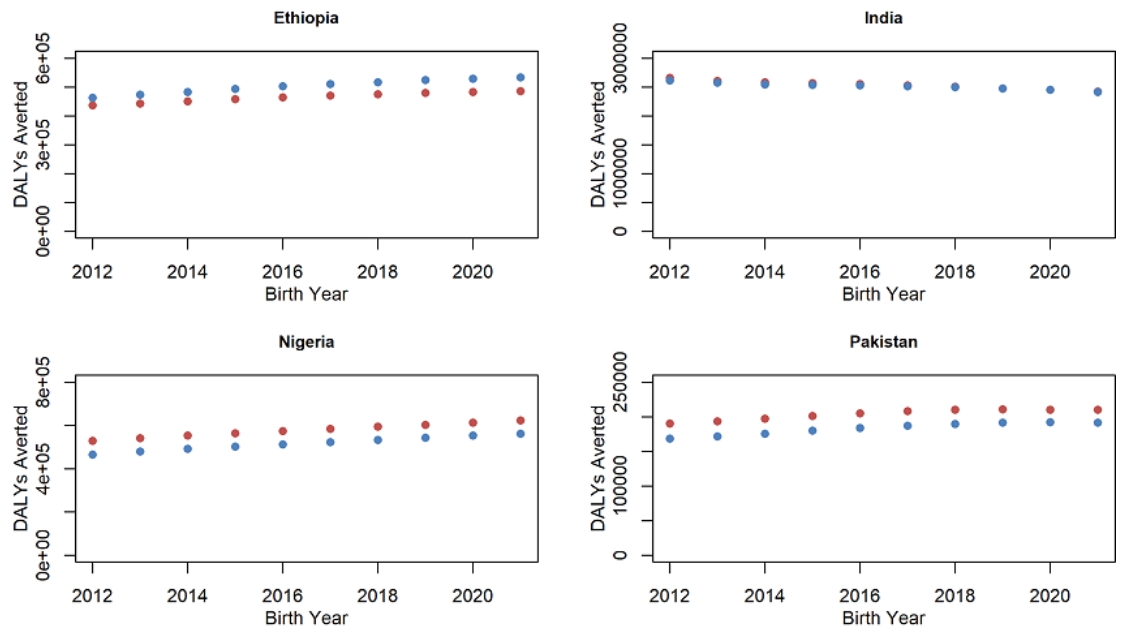

Note: Red dots $=$ Harvard; blue dots $=$ PRIME .

Figure 2 Cervical cancer cases, deaths and DALYs averted among girls vaccinated during 2021-2030 by country since time of vaccination until 2100: (A) cases averted; (B) deaths averted; (C) DALYs averted. DALYs, disability-adjusted life years; PRIME, Papillomavirus Rapid Interface for Modelling and Economics.

Similarly, the Harvard model estimated a greater number of cervical cancer deaths averted than the PRIME model by 15\% in Ethiopia, 17\% in India, 19\% in Nigeria and 24\% in Pakistan (figure 2B). Specifically, the estimated number of cervical cancer deaths averted ranged from 210000 to 248000 in Ethiopia; 1130000 
A

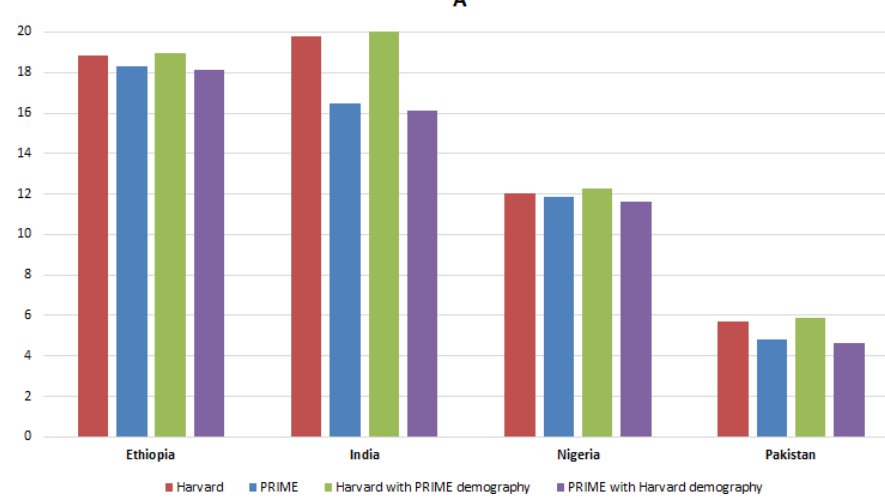

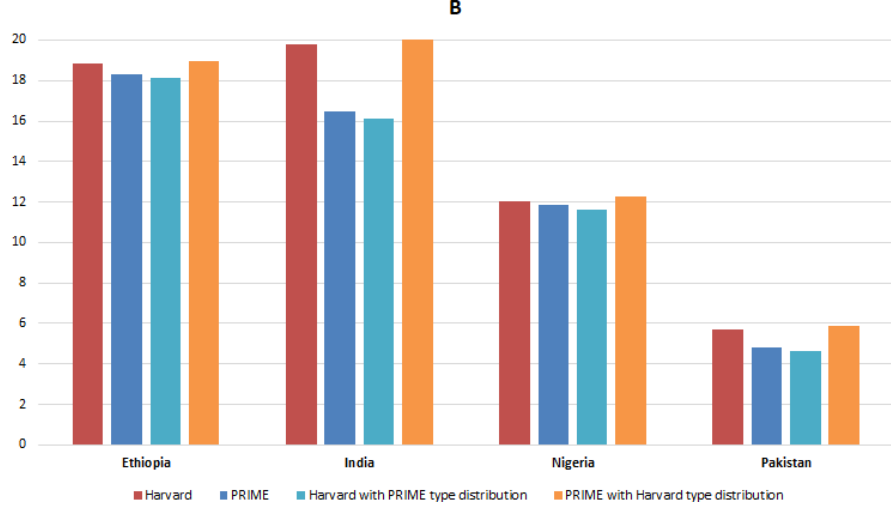

Figure 3 Cervical cancer cases averted per 1000 fully vaccinated girls for cohorts vaccinated during 2021-2030 since time of vaccination to 2100: (A) with alignment on population demography; and (B) with alignment on HPV 16/18-type distribution. HPV, human papillomavirus; PRIME, Papillomavirus Rapid Interface for Modelling and Economics.

to 1350000 in India; 254000 to 314000 in Nigeria and 77200 to 102000 in Pakistan in the PRIME and Harvard models, respectively.

However, the PRIME model estimated a greater number of DALYs averted than the Harvard model by $8 \%$ in Ethiopia, whereas the Harvard model estimated a greater number of DALYs averted than the PRIME model by $1 \%$ in India, $11 \%$ in Nigeria and $10 \%$ in Pakistan (figure 2C). Specifically, the range between the PRIME model and the Harvard model for the estimated number of cervical cancer DALYs averted was: 4650000 to 5030000 in Ethiopia; 25200000 to 25400000 in India; 5160000 to 5770000 in Nigeria and 1830000 to 2040000 in Pakistan.

\section{Vaccination impact}

Figure 3 shows the number of cervical cancer cases prevented per 1000 fully vaccinated girls in each of the four countries compared using both Harvard and PRIME models. When comparing the models under the respective model assumptions for HPV-type distribution and population demography, the estimated vaccination impact per 1000 fully vaccinated girls for the Harvard and PRIME models, respectively, was 19 versus 18 in Ethiopia; 20 versus 16 in India; 12 versus 12 in Nigeria and 6 versus 5 in Pakistan. When harmonising the assumptions around population demography across the Harvard and PRIME models, the difference in the estimated number of cervical cancer cases given routine vaccination narrowed, but the difference in the estimated vaccination impact was slightly increased (figure 3A). However, overall, the effect of harmonising the assumptions around population demography between Harvard and PRIME was small based on this metric, resulting in an equivalent estimate of the number of cervical cancer cases averted per 1000 fully vaccinated girls as the base case Harvard and PRIME models. On the other hand, when harmonising the assumptions around HPV-16/18-type distribution between the Harvard and PRIME models (figure 3B), the differences in estimated vaccination impact were nearly eliminated. Thereby, we infer that the main difference in estimates for cases averted by vaccination between the two models is due to variations in cervical cancer attribution to HPV-16/18.

\section{DISCUSSION}

The Harvard and PRIME models are used by both VIMC and other global stakeholders to project the impact of HPV vaccination. The two models differ in their inputs and assumptions for HPV-16/18-type distribution, population demography, cervical cancer mortality and estimation of DALYs. The proportion of cervical cancers due to HPV-16/18 is relatively higher in the Harvard model, especially for India and Pakistan, and thereby cases averted (figure 2A) and vaccination impact (cases averted per 1000 fully vaccinated girls) are relatively higher (figure 3) in the Harvard model for India and Pakistan. The difference between these two models captures variation around interpretation of input data. In the case of HPV-16/18-type distribution, the Harvard model relied on a meta-analysis of cross-sectional high-risk HPVtype distribution in HPV-positive women, ${ }^{32}$ whereas the PRIME model relied on ${ }^{32}$ the study by Serrano et al. ${ }^{31}$ whose data sources included the same meta-analysis ${ }^{32}$ and a retrospective cross-sectional worldwide study, ${ }^{33}$ and accounts for multitype infections through proportional weighting attribution. ${ }^{31}$

HPV-16/18 vaccination was estimated to avert substantial numbers of cervical cancer cases, deaths and DALYs by both the Harvard model and the PRIME model. Both models provide similar results to previous analyses of vaccination impact in the analysed countries, including multicountry ${ }^{141517-214041}$ and single-country analyses, ${ }^{12} 42$ most of which used earlier versions of the Harvard and PRIME models.

However, routine HPV vaccination has yet to be introduced in any of these four high-burden countries at the national level (although a nationwide single-age cohort campaign of 14-year-old girls was conducted in Ethiopia in 2018-2019 $9^{12}$ and India introduced in a few states in $2016^{13}$ ). In order to accelerate progress towards cervical cancer elimination, preventing cervical cancer through HPV vaccination will be an essential strategy in these 
countries, particularly given the low coverage and access to cervical cancer screening. ${ }^{8}$ Understanding similarities and differences between HPV vaccination impact predicted by different models will be crucial, given that key questions about which countries to prioritise and which vaccination strategies to use will be important in an era of HPV vaccine dose shortages ${ }^{43}$ and COVID-19related disruptions to vaccination programmes. ${ }^{44}$

The size of the relevant population at each year of age is critical because it determines the number of people who are exposed to the risk of cervical cancer and can, therefore, be protected by vaccination. Both methods used to estimate the at-risk population have strengths and limitations. In the PRIME model, the size of the age cohort in subsequent years is calculated by constructing life tables, using the time-varying probability of dying by age and country from the World Population Prospects 2019 estimates. ${ }^{36}$ The Harvard method not only captures such changes but also includes future population change due to migration, which may not reflect the same vaccination status as the locally born population. In general, as the demography differences are minimal between the two models, the vaccination-impact estimates are similar for each country when demography is switched to align with the alternative model. The main driver of the differences in the vaccination impact was HPV-16/18-type distribution-or, the proportion of cervical cancers that can be averted by the bivalent vaccine, which has a direct relationship with vaccination impact. As cervical cancer deaths are directly estimated from cervical cancer cases, HPV-16/18-type distribution is likewise the main driver of the differences in vaccination impact in terms of cervical cancer deaths averted, with additional differences due to the mortality estimation approach: stage-specific (Harvard) versus age-specific (PRIME). The years lived with disability for the estimated cervical cancer cases and the years of life lost for the estimated cervical cancer deaths both contribute to the estimates of DALYs averted by HPV vaccine, and, therefore, are likewise driven by differences in HPV-16/18-type distribution. However, the disability weights assumed by the Harvard and PRIME models differ and contribute to additional differences.

This comparative modelling exercise highlighted differences in the estimates of health impact of HPV vaccination due to model uncertainty. We note that HPV vaccine projections are particularly susceptible to large swings in estimated health outcomes due to even small changes in fertility and mortality because of the long time horizons needed in the models. Comparative modelling exercises as we have done can enhance model transparency and clarify the range of uncertainty in vaccine impact. Hence, the differences between the models are a strength that reflects the variation and uncertainty in the projected health outcomes of vaccination impact. Understanding the intermodel variation improves the quality and coordination of vaccine-impact assessment, which in turn can help guide public health research and priorities in cervical cancer elimination and control.
Similar comparative modelling exercises were conducted to examine the timeline to cervical cancer elimination in LMICs. ${ }^{20}{ }^{21}$ Relying on evidence synthesis from different models was deemed an essential aspect to inform strategies for cervical cancer elimination by the WHO. ${ }^{538}$ However, these analyses relied on estimations of age-standardised cervical cancer incidence, such that demographic changes were not expected to drive the uncertainty in the timing of elimination.

There are several important limitations to this analysis. As we relied on static cohort models in these analyses, we were only able to estimate direct effects for vaccinated women, which excluded additional indirect benefits from herd immunity for unvaccinated women. We projected intervention impact for only 10 cohorts of 9-year-old girls in four countries and assumed that cervical cancer incidence rates affecting these cohorts would be stable over the time period of the analysis. Vaccine efficacy against high-risk HPV types other than HPV-16/18 (ie, cross-protection) was not included. We did not examine cervical cancer screening programmes in this analysis and assumed that any ongoing screening programmes did not change as HPV vaccination introduction and delivery changed. Additionally, given limited data on the burden of other HPV-related diseases in LMICs, we did not evaluate the impact HPV vaccination may have on non-cervical cancers in women and men.

\section{CONCLUSION}

Both models project that HPV vaccination will have a large impact on morbidity and mortality in the four countries we examined. The differences in outcomes between the models capture variation in interpretation around data on cervical cancer epidemiology and future demographic change. This study highlights that HPV-type distribution is a critical input to modelling the potential health impact of vaccination. The main difference in estimates for cases and deaths averted by vaccination between the models capture variation in interpretation around data on cervical cancer attribution to HPV-16/18. The main differences in estimates for DALYs averted by vaccination between the models are due to variations in cervical cancer attribution to HPV-16/18, disability weights, and age-specific life expectancy. Continued explorations of underlying differences in model inputs, assumptions, and results will be crucial when examining public health policy.

\section{Twitter Allison Portnoy @AllisonPortnoy and Mark Jit @markjit}

Contributors AP and KA conducted the data analysis and drafted the manuscript with input from all authors. All authors conceptualised the study and approved the final version of the manuscript. AP accepts full responsibility for the finished work and/or the conduct of the study, had access to the data, and controlled the decision to publish.

Funding This work was carried out as part of the Vaccine Impact Modelling Consortium (www.vaccineimpact.org), but the views expressed are those of the authors and not necessarily those of the Consortium or its funders. The funders were given the opportunity to review this paper prior to publication, but the final decision on the content of the publication was taken by the authors. This work 
was supported, in whole or in part, by the Bill \& Melinda Gates Foundation, via the Vaccine Impact Modelling Consortium [grant number INV-009125]. Under the grant conditions of the Foundation, a Creative Commons Attribution 4.0 Generic License has already been assigned to the Author Accepted Manuscript version that might arise from this submission.

\section{Competing interests None declared.}

Patient and public involvement Patients and/or the public were not involved in the design, or conduct, or reporting, or dissemination plans of this research.

\section{Patient consent for publication Not applicable.}

Provenance and peer review Not commissioned; externally peer reviewed.

Data availability statement Data are available upon reasonable request. The datasets generated during and/or analysed during the current study are available from the corresponding author on reasonable request.

Open access This is an open access article distributed in accordance with the Creative Commons Attribution 4.0 Unported (CC BY 4.0) license, which permits others to copy, redistribute, remix, transform and build upon this work for any purpose, provided the original work is properly cited, a link to the licence is given, and indication of whether changes were made. See: https://creativecommons.org/ licenses/by/4.0/.

\section{ORCID iDs}

Allison Portnoy http://orcid org/0000-0002-8467-1324

Kaja Abbas http://orcid.org/0000-0003-0563-1576

Mark Jit http://orcid.org/0000-0001-6658-8255

\section{REFERENCES}

1 Forman D, de Martel C, Lacey CJ, et al. Global burden of human papillomavirus and related diseases. Vaccine 2012;30 Suppl 5:F12-23.

2 Muñoz N, Bosch FX, de Sanjosé S, et al. Epidemiologic classification of human papillomavirus types associated with cervical cancer. $N$ Engl J Med 2003;348:518-27.

3 Garland SM, Hernandez-Avila M, Wheeler CM, et al. Quadrivalent vaccine against human papillomavirus to prevent anogenital diseases. N Engl J Med 2007;356:1928-43.

4 Hildesheim A, Wacholder S, Catteau G, et al. Efficacy of the HPV$16 / 18$ vaccine: final according to protocol results from the blinded phase of the randomized Costa Rica HPV-16/18 vaccine trial. Vaccine 2014;32:5087-97.

5 Brisson M, Drolet M. Global elimination of cervical cancer as a public health problem. Lancet Oncol 2019;20:319-21.

6 World Health Organization. Accelerating cervical cancer elimination. Report by the director-general, 2018. Available: http://apps.who.int/ gb/ebwha/pdf_files/EB144/B144_28-en.pdf

7 World Health Organization. Cervical cancer elimination strategy, 2019. Available: https://www.who.int/initiatives/cervical-cancerelimination-initiative

8 Bruni L, Saura-Lázaro A, Montoliu A, et al. Hpv vaccination introduction worldwide and who and UNICEF estimates of national HPV immunization coverage 2010-2019. Prev Med 2021;144:106399.

9 Wittet S, Tsu V. Cervical cancer prevention and the millennium development goals. Bull World Health Organ 2008;86:488-90.

10 Parkin DM, Ferlay J, Jemal A. Cancer in sub-Saharan Africa. Lyon, France: International Agency for Research on Cancer, 2018.

11 International Agency for Research on Cancer. International agency for research on cancer: GLOBOCAN 2020. cancer incidence, mortality and prevalence worldwide. IARC cancer base: Lyon, France, 2020. Available: https://gco.iarc.fr/today/home

12 Portnoy A, Sweet S, Desalegn D, et al. Health gains and financial protection from human papillomavirus vaccination in Ethiopia: findings from a modelling study. Health Policy Plan 2021;36:891-9.

13 Sankaranarayanan R, Basu P, Kaur P, et al. Current status of human papillomavirus vaccination in India's cervical cancer prevention efforts. Lancet Oncol 2019;20:e637-44.

14 Lee LA, Franzel L, Atwell J, et al. The estimated mortality impact of vaccinations forecast to be administered during 2011-2020 in 73 countries supported by the GAVI alliance. Vaccine 2013;31 Suppl 2:B61-72

15 Li X, Mukandavire C, Cucunubá ZM, et al. Estimating the health impact of vaccination against ten pathogens in 98 low-income and middle-income countries from 2000 to 2030: a modelling study. Lancet 2021;397:398-408.
16 Jit M, Brisson M. Modelling the epidemiology of infectious diseases for decision analysis: a primer. Pharmacoeconomics 2011;29:371-86.

17 Goldie SJ, O'Shea M, Campos NG, et al. Health and economic outcomes of HPV 16,18 vaccination in 72 GAVI-eligible countries. Vaccine 2008;26:4080-93.

18 Jit M, Brisson M. Potential lives saved in 73 countries by adopting multi-cohort vaccination of 9-14-year-old girls against human papillomavirus. Int J Cancer 2018;143:317-23.

19 Burger EA, Portnoy A, Campos NG, et al. Choosing the optimal HPV vaccine: the health impact and economic value of the nonavalent and bivalent HPV vaccines in 48 Gavi-eligible countries. Int J Cancer 2021;148:932-40.

20 Brisson M, Kim JJ, Canfell K, et al. Impact of HPV vaccination and cervical screening on cervical cancer elimination: a comparative modelling analysis in 78 low-income and lower-middle-income countries. Lancet 2020;395:575-90.

21 Canfell K, Kim JJ, Brisson M, et al. Mortality impact of achieving WHO cervical cancer elimination targets: a comparative modelling analysis in 78 low-income and lower-middle-income countries. Lancet 2020;395:591-603.

22 Jit M, Brisson M, Portnoy A, et al. Cost-Effectiveness of female human papillomavirus vaccination in 179 countries: a PRIME modelling study. Lancet Glob Health 2014:2:e406-14.

23 Burger EA, Campos NG, Sy S, et al. Health and economic benefits of single-dose HPV vaccination in a Gavi-eligible country. Vaccine 2018;36:4823-9.

24 Van Minh H, My NTT, Jit M, . Cervical cancer treatment costs and cost-effectiveness analysis of human papillomavirus vaccination in Vietnam: a PRIME modeling study. BMC Health Serv Res 2017;17:353.

25 Abbas KM, van Zandvoort K, Brisson M, et al. Effects of updated demography, disability weights, and cervical cancer burden on estimates of human papillomavirus vaccination impact at the global, regional, and national levels: a PRIME modelling study. Lancet Glob Health 2020;8:e536-44.

26 FUTURE II Study Group. Quadrivalent vaccine against human papillomavirus to prevent high-grade cervical lesions. N Engl J Med 2007;356:1915-27.

27 Malagón T, Drolet M, Boily M-C, et al. Cross-Protective efficacy of two human papillomavirus vaccines: a systematic review and metaanalysis. Lancet Infect Dis 2012;12:781-9.

28 Naud PS, Roteli-Martins CM, De Carvalho NS, et al. Sustained efficacy, immunogenicity, and safety of the HPV-16/18 AS04adjuvanted vaccine: final analysis of a long-term follow-up study up to 9.4 years post-vaccination. Hum Vaccin Immunother 2014;10:2147-62.

29 Paavonen J, Naud P, Salmerón J, et al. Efficacy of human papillomavirus (HPV)-16/18 AS04-adjuvanted vaccine against cervical infection and precancer caused by oncogenic HPV types (PATRICIA): final analysis of a double-blind, randomised study in young women. Lancet 2009;374:301-14

30 Schiller JT, Castellsagué X, Garland SM. A review of clinical trials of human papillomavirus prophylactic vaccines. Vaccine 2012;30 Suppl 5:F123-38.

31 Serrano B, Alemany L, Tous S, et al. Potential impact of a nine-valent vaccine in human papillomavirus related cervical disease. Infect Agent Cancer 2012;7:38.

32 Guan P, Howell-Jones R, Li N, et al. Human papillomavirus types in 115,789 HPV-positive women: a meta-analysis from cervical infection to cancer. Int J Cancer 2012;131:2349-59.

33 de Sanjose S, Quint WG, Alemany L, et al. Human papillomavirus genotype attribution in invasive cervical cancer: a retrospective cross-sectional worldwide study. Lancet Oncol 2010;11:1048-56.

34 Vaccine Impact Modelling Consortium. General guidance for DALYs calculation, 2019. Available: https://www.vaccineimpact.org/ resources/VIMC-model-run-DALYs-guidance_20190917-121647d5d6ef09_report.pdf

35 Global Burden of Disease Collaborative Network. Global burden of disease study 2017 (GBD 2017) disability weights. Seattle, United States: Institute for Health Metrics and Evaluation (IHME), 2018.

36 United Nations: World Population Prospects. United Nations population division, 2019. Available: https://population.un.org/ wpp/

37 World Health Organization. Life tables for WHO member states, 2019. Available: https://apps.who.int/gho/data/node.main.687?lang= en

38 World Health Organization. Global strategy to accelerate the elimination of cervical cancer as a public health problem, 2020. Available: https://www.who.int/publications/i/item/9789240014107 
39 den Boon S, Jit M, Brisson M, et al. Guidelines for multi-model comparisons of the impact of infectious disease interventions. BMC Med 2019;17:163

40 Kim JJ, Campos NG, O'Shea M, et al. Model-Based impact and cost-effectiveness of cervical cancer prevention in sub-Saharan Africa. Vaccine 2013;31 Suppl 5:F60-72.

41 Toor J, Echeverria-Londono S, Li X, et al. Lives saved with vaccination for 10 pathogens across 112 countries in a preCOVID-19 world. eLife 2021;10:e67635. doi:10.7554/eLife.67635

42 Diaz M, Kim JJ, Albero G, et al. Health and economic impact of HPV 16 and 18 vaccination and cervical cancer screening in India. $\mathrm{Br} \mathrm{J}$ Cancer 2008;99:230-8.
43 World Health Organization. Global market study: HPV vaccines. Geneva: World Health Organization, 2019. https://apps.who.int/iris/ handle/10665/311275

44 World Health Organization. Pulse survey on continuity of essential health services during the COVID-19 pandemic, 2020. Available: https://www.who.int/publications/i/item/WHO-2019-nCoV-EHS_ continuity-survey-2020.1

45 World Health Organization. Immunization and COVID-19 - Second pulse poll offers a more detailed understanding of disruptions to vaccination caused by COVID-19 and how to respond, 2020. Available: https://www.who.int/publications/m/item/gin-june-2020 